\title{
Effect of conditioning and test stimulus intensity on cortical excitability using triad-conditioning transcranial magnetic stimulation
}

\author{
Shady Safwat Hassan ${ }^{1,2}$ (1) $\cdot$ Carlos Trenado ${ }^{1,3} \cdot$ Tarek Ali Rageh $^{2} \cdot$ Alfons Schnitzler $^{1,4} \cdot$ Stefan Jun Groiss ${ }^{1,4}$
}

Received: 2 December 2019 / Accepted: 13 April 2020 / Published online: 22 April 2020

(c) The Author(s) 2020

\begin{abstract}
Cortical facilitation assessed with triad conditioning transcranial magnetic stimulation has been termed triad-conditioned facilitation (TCF). TCF has been supposed to reflect increased intracortical facilitation (ICF) at short interstimulus intervals (ISI) around $10 \mathrm{~ms}$ and an intrinsic rhythm of the motor cortex at longer ISI around $25 \mathrm{~ms}$. To gain further insight into the pathophysiological mechanism of TCF, we systematically studied the effect of suprathreshold conditioning stimulus (CS) and test stimulus (TS) intensity on TCF. Various CS intensities and TS intensities were used in a triad-conditioning paradigm that was applied to 11 healthy subjects. ISI between pulses were studied between 5 and $200 \mathrm{~ms}$. TCF at $10 \mathrm{~ms}$ ISI enhanced with increasing CS intensity but decreased with increasing TS intensity. The duration of facilitation was longer with higher CS intensity. However, TCF at $25 \mathrm{~ms}$ ISI could not be elicited with none of the CS and TS intensities addressed here. Our results are consistent with the notion of TCF at short ISI reflecting ICF. The enhanced and prolonged facilitation with increase of CS without additional isolated facilitation at longer ISI suggest a prolongation of ICF.
\end{abstract}

Keywords Transcranial magnetic stimulation (TMS) $\cdot$ Motor-evoked potentials (MEP) · Triad-conditioning facilitation (TCF) $\cdot$ Conditioning stimulus (CS) $\cdot$ Test stimulus (TS) $\cdot$ Interstimulus intervals (ISI)

Communicated by John C. Rothwell.

Electronic supplementary material The online version of this article (https://doi.org/10.1007/s00221-020-05812-z) contains supplementary material, which is available to authorized users.

Stefan Jun Groiss

groiss@uni-duesseldorf.de

1 Institute of Clinical Neuroscience and Medical Psychology, Medical Faculty, Heinrich Heine University, Düsseldorf, Germany

2 Department of Neurology, Assiut University Hospital, Assiut, Egypt

3 Translational Neuromodulation Unit, Department of Psychology and Neurosciences, Leibniz Research Centre for Working Environment and Human Factors, Technical University Dortmund, Dortmund, Germany

4 Department of Neurology, Medical Faculty, Heinrich Heine University, Moorenstr 5, 40225 Düsseldorf, Germany

\section{Introduction}

Transcranial magnetic stimulation (TMS) provides a sensitive and non-invasive tool to modulate excitability of different components of neural tissues (Kobayashi and PascualLeone 2003). Various conditioned TMS paradigms have been reported using the paired-pulse technique. For instance, a suprathreshold test stimulus (TS) preceded by a subthreshold conditioning stimulus (CS) at very short interstimulus intervals (ISI) below $5 \mathrm{~ms}$ gives place to suppression of MEPs (Short-interval IntraCortical Inhibition $=$ SICI). On the other hand, when preceded by a subthreshold CS at longer ISIs, such as 10-15 ms, MEPs are facilitated (IntraCortical Facilitation = ICF) (Kujirai 1993; Ziemann 1996; Ziemann and Chapter 2003; Hanajima 2002).

Hanajima et al. reported a new triad-conditioning method to study the modulation of motor cortical excitability in response to rhythmic stimulation of M1 (Hanajima (2009)). They used three monophasic TMS pulses over M1 applied at certain frequency in terms of rhythmic conditioning stimulation and found facilitation of the MEP to TS at an ISI around $10 \mathrm{~ms}$ and $25 \mathrm{~ms}$, which was termed triad conditioned facilitation (TCF). TCF has been supposed to reflect increased 
ICF at short ISI around $10 \mathrm{~ms}$ and intrinsic rhythm of the motor cortex at longer ISI around $25 \mathrm{~ms}$. Noteworthy, alteration of TCF at $25 \mathrm{~ms}$ has been shown in several diseases like cortical myoclonus, Parkinson's disease or amyotrophic lateral sclerosis (Hanajima 2011, 2014; Groiss 2017). In Hanajima's first report (2009), the effect of varying CS intensity showed that a specific combination of CS [intensity $110 \%$ of active motor threshold (AMT), ISI $=25 \mathrm{~m}$ ] is required to evoke TCF (Hanajima 2009). However, the effect of CS with higher intensity or variation of the TS intensity have not been investigated so far. The aim of this study was to probe the effect of variable CS and TS intensities on TCF to shed further light on its pathophysiological mechanism.

\section{Materials and methods}

\section{Subjects}

Eleven healthy volunteers (eight men and three women, range 22-45 years, mean 28.1, SD 7.4) participated in the study after giving written informed consent. No subject had neurological, psychiatric, or other medical problems or any contraindication to TMS (Rossi (2009)). The experiment was performed according to the Declaration of Helsinki and was approved by the Ethics Committee of the University of Düsseldorf, Germany (5738R).

\section{Electromyography (EMG) recordings}

Surface electromyography (EMG) signals were recorded from the right first dorsal interosseous (FDI) muscle in a belly tendon montage using 9-mm diameter $\mathrm{Ag}-\mathrm{AgCl}$ surface cup electrodes. Responses were amplified (Digitimer D360, UK) and filtered (100-5000 Hz), digitized at a sampling rate of $5 \mathrm{kHz}$, and stored on a computer that was used to perform the off-line analysis. Subjects were instructed to keep the right FDI relaxed throughout the experiment, which was monitored online with an oscilloscope.

\section{Transcranial magnetic stimulation}

Magstim $200^{2}$ magnetic stimulators (The Magstim Company Ltd., UK) were used to deliver TMS. Four magnetic stimulators were connected with a specially designed combining module (The Magstim Co. Ltd., Whitland, UK) to allow the application of up to four monophasic magnetic stimuli through a single figure-of-eight shaped coil with an outer diameter of $7 \mathrm{~cm}$ at each wing (Hanajima 2009; Groiss 2017, 2013). Left M1 was chosen as stimulation site. First, the hotspot for the right FDI was identified. Subsequently, resting motor threshold (RMT) and AMT were precisely determined. RMT was defined as the lowest stimulator output intensity capable of eliciting MEPs of $50 \mu \mathrm{V}$ peak-to-peak amplitude in the relaxed FDI muscle in more than 5 of 10 consecutive trials (Rossini et al. 2015). AMT was defined as the lowest stimulation intensity that still evoked small responses of $100 \mu \mathrm{V}$ amplitudes in half of the trials during slight voluntary contraction (approx. 5-10\% of maximal contraction) (Groiss 2017; Rothwell et al. 1999). In case of presence of a clear silent period of any duration, smaller amplitudes of $50 \mu \mathrm{V}$ were also regarded as response.

\section{Triad conditioned paradigm}

The original protocol of the triad conditioning paradigm consisted of a suprathreshold TS preceded by three monophasic CS with varying ISI. The intensity of the CS was set to $110 \%$ of AMT and the intensity of the TS was set to elicit MEPs of about $0.3 \mathrm{mV}$ (Hanajima 2009). In this study, we performed two blocks of trials for each subject to investigate the effect of various TS and CS intensities. In the first block, the intensity of the CS was fixed to $110 \%$ AMT and we applied 3 different TS intensities according to the RMT (100\% RMT, 120\% RMT and 140\% RMT), we named this block TS block. In the second block, we fixed the TS intensity at $120 \%$ RMT and used variable CS intensities according to the AMT (110\% AMT, 120\% AMT and 130\% AMT), so this block was named CS block. The ISIs were varied between 5 and $200 \mathrm{~ms}$ resulting in 10 conditions ( 5 , $10,12.5,20,25,40,50,100,200 \mathrm{~ms}$ which corresponds to the following frequencies in order $(200,100,80,66,50$, $40,25,20,10,5 \mathrm{~Hz}$ ) and one control condition (for the test stimulus alone) and each condition was applied in a shuffled randomized order. Compared to the original report, the number of conditions were reduced to allow the measurements to be done in one session.

\section{Statistical and data analysis}

Statistical analysis was performed using GraphPad Prism (GraphPad Software, CA, USA) and IBM SPSS Statistics (Version 24, IBM Software, Business and analytics, Armonk, NY, USA). We used two-way repeated measures ANOVA with within-factor ISI and between factor AMTand RMT- intensities for each block to compare triad-conditioned MEPs. Post hoc Bonferroni tests were performed whenever an interaction was found. One-way ANOVA with post hoc Dunnett's test was done in each group to compare the degree of facilitation at each ISI with the baseline. To determine a prospective relationship between the degree of facilitation at each ISI and MEP amplitudes at TS intensity (MSO\%), correlation analysis with the Holm correction for multiple comparisons was performed [by considering parameters $m=18$ (number of $p$ values) and threshold $* p=0.003$ similarly as specified in (Trenado et al. 2018)]. 


\section{Results}

Figure $1 \mathrm{a}, \mathrm{b}$ shows MEP size ratios as a function of ISI for the different TS and CS intensity settings. Two-way repeated measures ANOVA showed significant effect of ISI and interaction between ISI and stimulus intensity for both blocks. For TS block [ISI: $F(8,240)=21.75$, $p<0.0001$; test intensity: $F(2,240)=2.19, p=0.12$; interaction (ISI $\times$ test intensity): $F(16,240)=4.7, p<0.0001$ ], post hoc Bonferroni test revealed significant differences in MEP amplitudes between TS intensities 100\% RMT and $120 \%$ RMT at ISI $10 \mathrm{~ms}$ and between $100 \%$ RMT and $140 \%$ RMT at ISI 5, 10 and 12.5 ms. For CS block [ISI: $F(8,240)=27.9, p<0.0001$; test intensity: $F$ $(2,240)=1.16, p=0.37$; interaction (ISI $\times$ test intensity): $F(16,240)=3.72, p<0.0001]$, post hoc Bonferroni test showed significant differences in MEP amplitudes between CS intensity $110 \%$ AMT and 130\% AMT at ISI 10, 12.5 and $20 \mathrm{~ms}$ (Fig. 1c, d). Compared to baseline one-way ANOVA with post hoc Dunnett's test revealed the following results: For $100 \%$ RMT intensity there was significant MEP facilitation at 5, 10, $12.5 \mathrm{~ms}$, while in $120 \%$ RMT and $140 \%$ RMT facilitation was significant at 10 and $12.5 \mathrm{~ms}$. In the CS block there was significant facilitation for $110 \% \mathrm{AMT}$ at 10 and $12.5 \mathrm{~ms}$ and for $120 \% \mathrm{AMT}$ and $130 \%$ AMT at 10, 12.5 and $20 \mathrm{~ms}$ in comparison to the baseline. Figure 2 shows significant negative correlation between degree of MEP facilitation at $12.5 \mathrm{~ms}$ and single pulse MEP amplitude $(r=-0.53, p=0.002)$.

The following TMS characteristics were found in the current study: Thresholds for the study participants [RMT $(54.3 \% \pm 8.3)$, AMT $(35.5 \pm 5.8)]$; unconditioned MEP amplitudes for each TS intensity (100\% RMT, 120\% RMT and $140 \%$ RMT) across the different CS conditions were as follows: $0.3,1.01$ and $2.1 \mathrm{mV}$, respectively. It is worth to emphasize that a TS intensity of $100 \%$ RMT in our study was the most comparable condition to the criterion set by Hanajima et al. of using a TS intensity of $0.3 \mathrm{mV}$.

\section{Discussion}

In this study, we confirmed two main results. First, the triad conditioning TMS induced MEP facilitation at 10 and $12.5 \mathrm{~ms}$ that was enhanced with increasing CS intensity but decreased with increasing TS intensity, which supports the notion of TCF at short ISI sharing the same mechanisms as ICF (Kujirai 1993; Daskalakis 2004). Second, the

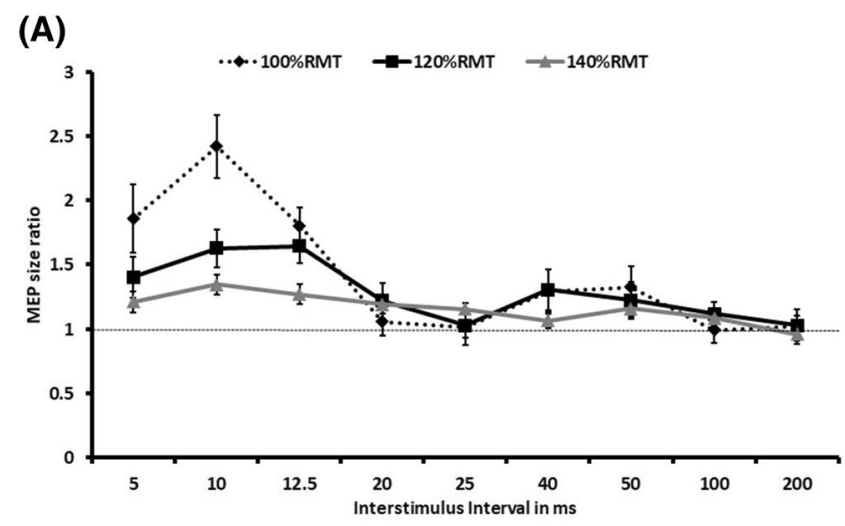

(B)
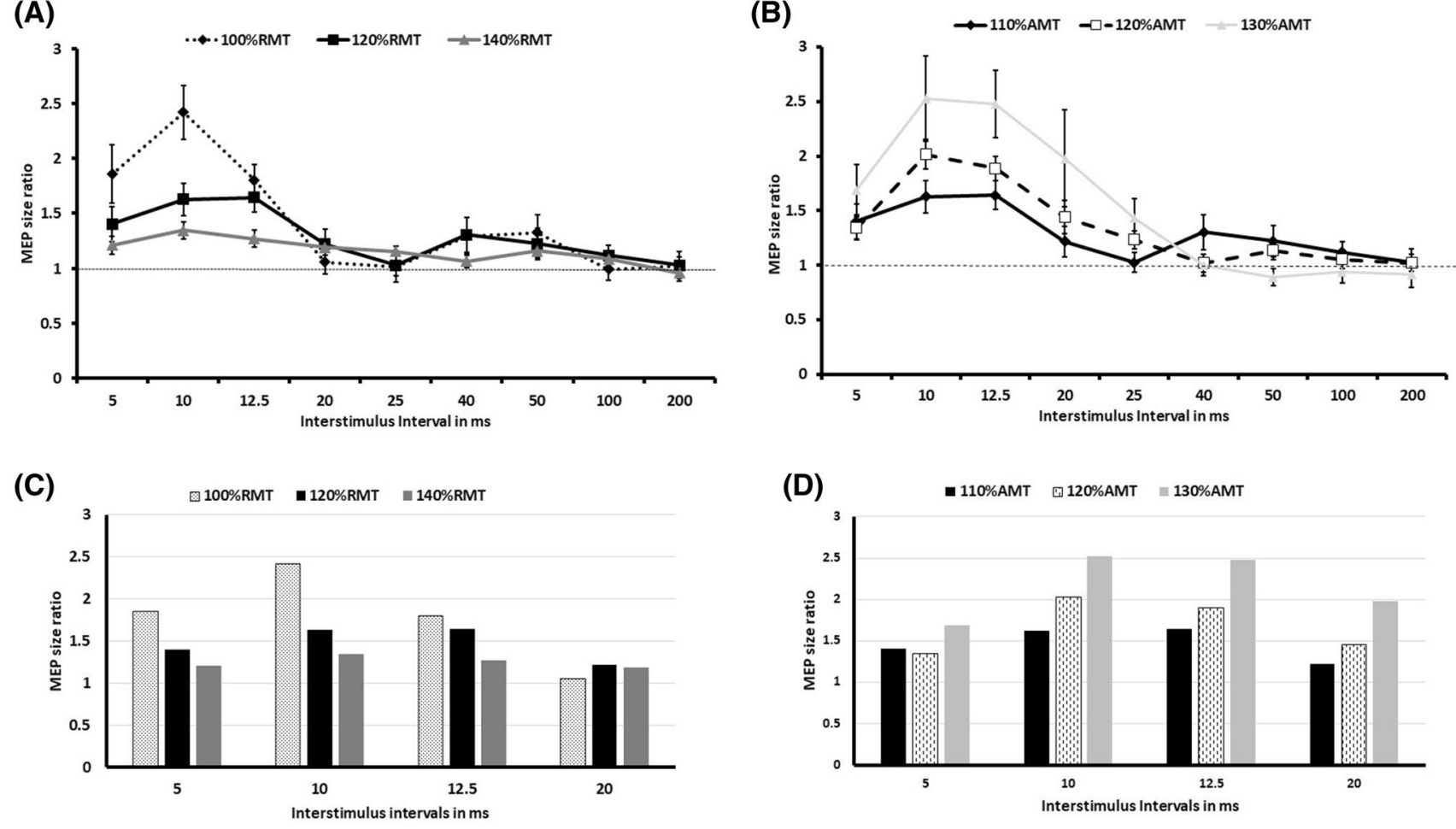

Fig. 1 a TS block results. Here, we show TCF results across ISIs under by varying of the test stimulus intensities; $\mathbf{b}$ CS block results. Here, it is shown TCF results across ISISs by varying intensities of

the conditioning stimuli; $\mathbf{c}, \mathbf{d}$ display bar graphs describing the comparison between different TS and CS intensities in the ISI between 5 and $20 \mathrm{~ms}$ 


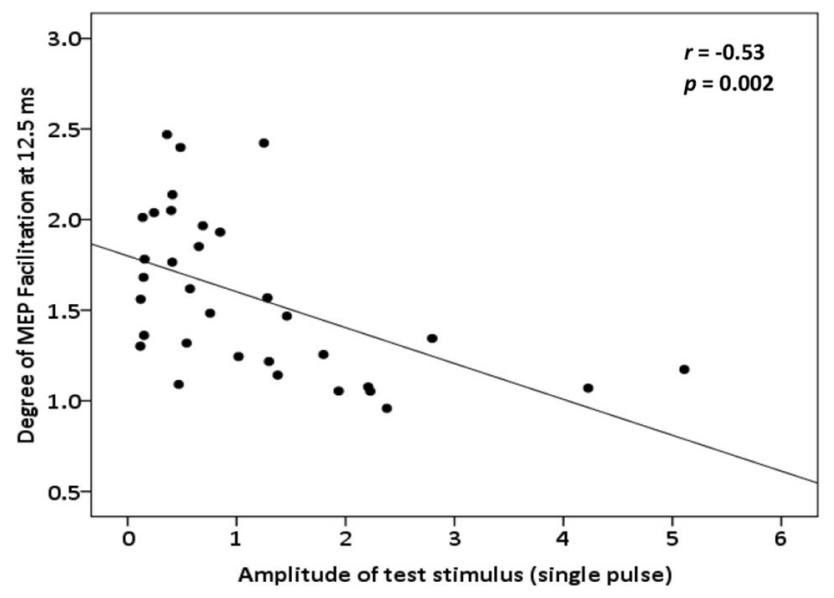

Fig. 2 Correlation analysis between MEP amplitude at TS intensity and degree of facilitation at $12.5 \mathrm{~ms}$. Insets illustrate the results of the linear correlation analysis (black line)

duration of TCF at shorter ISI became longer as CS intensity increased but isolated TCF at $25 \mathrm{~ms}$ was lacking with the addressed intensities here. This suggest a prolongation of ICF with CS intensity increase rather than a potentiation of intrinsic cortical rhythms.

In our study, when we increased intensities of CS (110\%AMT, 120\%AMT and 130\%AMT) preceding a 120\% RMT TS intensity, a gradual increase of facilitation occurred at short intervals around $10 \mathrm{~ms}$ and lasting up to $20 \mathrm{~ms}$ with lack of facilitation at $25 \mathrm{~ms}$. So, we found highest facilitation using the maximum conditioning subthreshold intensity (130\%AMT). On the other hand, facilitation decreased when we used a gradually increased intensity of the test stimulus following a CS at $110 \%$ AMT. In summary, facilitation was enhanced with increasing CS intensity and reduced with increasing TS intensity. This could be explained by the fact that higher intensity of TS may result in larger recruitment of interneurons making synaptic connections to corticospinal neurons and lower threshold interneurons responding with weaker intensity. Thus, reduction of TCF with higher intensity of TS might indicate less influence of conditioning stimuli. Accordingly, this will be in line with intensity dependent changes of ICF which have been described in paired-pulse TMS paradigms and, therefore, supports the hypothesis of TCF around $10 \mathrm{~ms}$ sharing the same mechanism as ICF (Kujirai 1993; Ziemann and Chapter 2003). These findings were confirmed by the correlation analysis, where the degree of facilitation at $12.5 \mathrm{~ms}$ ISI inversely correlated with the MEP amplitude at TS intensity. At the same time, we noticed prolonged duration of facilitation with increased intensities of both CS and TS.

The original experiments designed by Hanajima et al. (2009), reported incidence of MEP facilitation at two peaks. The first peak at ISI around $10 \mathrm{~ms}$ which is compatible with
ICF and the second termed TCF and occurring at ISI $25 \mathrm{~ms}$ which is suggested to represent increased motor cortical responsiveness to rhythmic external stimuli that is induced by an intrinsic $40 \mathrm{~Hz}$ rhythm of the motor cortex (Hanajima 2009). Owing to the relevance of the TCF at $25 \mathrm{~ms}$ for differentiation or diagnostics of neurological diseases as reported by previous clinical studies, we aimed to probe the effect of variable CS and TS intensities on TCF to shed further light on its pathophysiological mechanism. Interestingly, TCF at $25 \mathrm{~ms}$ was not elicited in our case. Several possibilities for explanation need to be taken into account. Given the fact that duration of ICF (in terms of TCF at short ISI) was prolonged by increasing CS without additional isolated TCF at longer ISI, facilitation at $25 \mathrm{~ms}$ may also reflect prolongated ICF, which would argue against the rhythm hypothesis for TCF $25 \mathrm{~ms}$. However, it is crucial to emphasize that there were methodological differences regarding our TMS settings and the previous studies. First of all, in the original paper, a coil orientation to induce PA currents in the brain with the coil handle in parallel to the midline was used. In our case, we also used PA currents but with coil handle oriented 45 degrees from the midline. The relevance of this aspect has been emphasized by previous studies reporting on changes in amplitude of MEP output as a function of coil orientation (Mills et al. 1992). However, since we were able to induce TCF $25 \mathrm{~ms}$ in our previously published paper, where we used the same current direction, the coil orientation may less likely be the reason for the differing results (Groiss et al. 2017). However, we believe that another prospective analysis comparing different coil directions will be necessary in the future to clarify this point.

Second, in the original reports, TS intensity was chosen based on the MEP amplitude (the intensity which elicit MEPs of about $0.3 \mathrm{mv}$ when given alone) and fixed the CS intensity to $110 \%$ AMT (Hanajima et al. 2009). In our study we adjusted our TS intensity referring to RMT and not to the MEP amplitude. However, single pulse TMS in the $100 \%$ RMT condition in fact elicited MEP responses of $0.27 \mathrm{mV}$ in average after omitting the trials without response in our study. This makes this condition likely comparable with the original reports. Finally, anatomical differences (e.g., the thickness and shape of skull) between participants in our study (which mainly considered subjects of European descent) and the previous studies, which considered Asian participants may be of relevance. Thus, ethnicity may represent a contributing factor to variability of cortical excitability in different populations due to anatomical differences as emphasized by earlier studies (Ball 2010).

Limitations of all studies on TCF include the rather small sample sizes, which further motivates multicenter studies to better understand the variability of TCF in healthy populations. 


\section{Conclusions}

Taken together, facilitation around $10 \mathrm{~ms}$ enhances with increasing CS intensity but decreases with increasing TS intensity. However, increasing CS intensity prolongs the duration of facilitation, while TCF at $25 \mathrm{~ms}$ could not be elicited. Our results are consistent with the notion of TCF at short ISI reflecting ICF. The increased duration of ICF with increase of CS without isolated TCF at longer ISI suggest a prolongation of ICF for TCF $25 \mathrm{~ms}$ as well and speaks against the rhythm hypothesis. The present results question the currently assumed hypothesis on TCF mechanisms and may be relevant regarding the optimization of triad conditioning paradigms for future clinical studies.

Acknowledgements Open Access funding provided by Projekt DEAL.

Funding Deutsche Forschungsgemeinschaft (Grant No. SFB 974) and Deutscher Akademischer Austauschdienst (Grant No. 57030312).

\section{Compliance with ethical standards}

Conflict of interest SSH was supported by the German Research Foundation through SFB 974, the German Academic Exchange Service and the Egyptian Ministry of Higher Education and Scientific Research. CT, TR, AS and SJG declare no conflicts of interest related to the current study.

Open Access This article is licensed under a Creative Commons Attribution 4.0 International License, which permits use, sharing, adaptation, distribution and reproduction in any medium or format, as long as you give appropriate credit to the original author(s) and the source, provide a link to the Creative Commons licence, and indicate if changes were made. The images or other third party material in this article are included in the article's Creative Commons licence, unless indicated otherwise in a credit line to the material. If material is not included in the article's Creative Commons licence and your intended use is not permitted by statutory regulation or exceeds the permitted use, you will need to obtain permission directly from the copyright holder. To view a copy of this licence, visit http://creativecommons.org/licenses/by/4.0/.

\section{References}

Ball R et al (2010) A comparison between Chinese and Caucasian head shapes. Appl Ergon 41(6):832-839
Daskalakis ZJ et al (2004) Exploring the connectivity between the cerebellum and motor cortex in humans. J Physiol 557(Pt 2):689-700

Groiss SJ et al (2013) Quadri-pulse stimulation induces stimulation frequency dependent cortical hemoglobin concentration changes within the ipsilateral motor cortical network. Brain Stimul 6(1):40-48

Groiss SJ et al (2017) Impairment of triad conditioned facilitation in amyotrophic lateral sclerosis. Amyotroph Lateral Scler Frontotemporal Degener 18(7-8):604-610

Hanajima R et al (2002) Mechanisms of intracortical I-wave facilitation elicited with paired-pulse magnetic stimulation in humans. J Physiol 538(Pt 1):253-261

Hanajima R et al (2009) Forty-hertz triple-pulse stimulation induces motor cortical facilitation in humans. Brain Res 1296:15-23

Hanajima R et al (2011) Triad stimulation frequency for cortical facilitation in cortical myoclonus. Mov Disord 26(4):685-690

Hanajima $R$ et al (2014) Triad-conditioning transcranial magnetic stimulation in Parkinson's disease. Brain Stimul 7(1):74-79

Kobayashi M, Pascual-Leone A (2003) Transcranial magnetic stimulation in neurology. Lancet Neurol 2(3):145-156

Kujirai T et al (1993) Corticocortical inhibition in human motor cortex. J Physiol 471:501-519

Mills KR, Boniface SJ, Schubert M (1992) Magnetic brain stimulation with a double coil: the importance of coil orientation. Electroencephalogr Clin Neurophysiol Evoked Potentials Sect 85(1):17-21

Rossi S et al (2009) Safety, ethical considerations, and application guidelines for the use of transcranial magnetic stimulation in clinical practice and research. Clin Neurophysiol 120(12):2008-2039

Rossini PM et al (2015) Non-invasive electrical and magnetic stimulation of the brain, spinal cord, roots and peripheral nerves: Basic principles and procedures for routine clinical and research application. An updated report from an IFCN Committee. Clin Neurophysiol 126(6):1071-1107

Rothwell JC et al (1999) Magnetic stimulation: motor evoked potentials. The International Federation of Clinical Neurophysiology. Electroencephalogr Clin Neurophysiol Suppl 52:97-103

Trenado C, Boschheidgen M, Rübenach J, N'Diaye K, Schnitzler A, Mallet L, Wojtecki L (2018) Assessment of metacognition and reversal learning in parkinson's disease: preliminary results. Front Hum Neurosci 12:343. https://doi.org/10.3389/fnhum.2018.00343

Ziemann U et al (1996) Effects of antiepileptic drugs on motor cortex excitability in humans: a transcranial magnetic stimulation study. Ann Neurol 40(3):367-378

Ziemann U (2003) Chapter 23 Pharmacology of TMS. In: Paulus W, et al (eds) Supplements to clinical neurophysiology. Elsevier, New York, pp 226-231

Publisher's Note Springer Nature remains neutral with regard to jurisdictional claims in published maps and institutional affiliations. 